\title{
Long-Term Efficacy of Edaravone in Patients With Acute Myocardial Infarction
}

\author{
Kenichi Tsujita, MD; Hideki Shimomura, MD**; Koichi Kaikita, MD; \\ Hiroaki Kawano, MD*; Jun Hokamaki, MD; Yasuhiro Nagayoshi, MD; \\ Takuro Yamashita, MD; Masaya Fukuda, MD; \\ Yoshinori Nakamura, MD**; Tomohiro Sakamoto, MD; \\ Michihiro Yoshimura, MD; Hisao Ogawa, MD
}

\begin{abstract}
Background The effect of edaravone, a free radical scavenger, on long-term prognosis and its efficacy with regards to scavenging injurious free radicals in patients with acute myocardial infarction (AMI) was examined. Methods and Results One hundred and one initial AMI patients were randomly assigned to receive $30 \mathrm{mg}$ edaravone $(n=50)$ or a placebo $(n=51)$ intravenously just before reperfusion. The infarct size, using serum biomarkers and Q-wave formations, and the incidence of reperfusion arrhythmia between the groups were compared. Cardiovascular event-free curves were estimated by using the Kaplan-Meier method. In addition, the serum thioredoxin levels, an oxidative stress marker, to assess the antioxidant effect of edaravone was determined. In all cases, successful reperfusion was obtained within $6 \mathrm{~h}$ after the onset of symptoms. Infarct size and reperfusion arrhythmia were significantly attenuated in the edaravone group compared with the placebo group $(\mathrm{p}=0.035$ and $\mathrm{p}=0.031$ ). The cumulative event-free rate was significantly higher in the edaravone group than in the placebo group ( $\mathrm{p}=0.045$ ). Serum thioredoxin levels were significantly lower in the edaravone group than in the placebo group throughout the acute phase.
\end{abstract}

Conclusions The present study suggests that the edaravone administration just prior to reperfusion might reduce oxidative stress and improve the long-term clinical outcomes of AMI patients. (Circ J 2006; 70: 832-837)

Key Words: Antioxidants; Free radicals; Myocardial infarction; Prognosis; Reperfusion

M yocardial reperfusion is necessary for the salvage and recovery of the ischemic myocardium. Early reperfusion therapy has improved the clinical outcomes of patients with acute myocardial infarction (AMI), but these benefits are limited in some patients by reperfusion injuries! There is now increasing evidence that reactive oxygen species (ROS) lead to reperfusion injuries in the ischemic myocardium and that they are related to subsequent left ventricular (LV) dysfunction, ${ }^{2,3}$ Recent investigations have shown that increased vascular oxidative stress predicts the risk of cardiovascular events in patients with coronary artery disease 4

Various different radical scavengers and antioxidants have been shown to effectively reduce reperfusion injury in experimental models 5,6 In these ischemia-reperfusion models, it was demonstrated that radical scavengers such as superoxide dismutase and vitamin $\mathrm{E}$ analog reduced infarct size by limiting reperfusion-induced oxidative stress and by attenuating the inflammatory response. On the basis of this established scientific rationale, several randomized controlled trials were designed to prove or disprove the causa-

(Received February 20, 2006; revised manuscript received April 21, 2006; accepted May 1, 2006)

Department of Cardiovascular Medicine, *Division of Intensive Care Unit, Graduate School of Medical Sciences, Kumamoto University, Kumamoto and **Department of Cardiology, Fukuoka Tokushukai Medical Center, Kasuga, Japan

Mailing address: Kenichi Tsujita, MD, Department of Cardiovascular Medicine, Graduate School of Medical Sciences, Kumamoto University, 1-1-1 Honjo, Kumamoto 860-8556, Japan. E-mail: tsujita@ kumamoto-u.ac.jp tive effects for antioxidant supplements?-10 Unfortunately, results of these studies on antioxidants and cardiovascular event risks have been disappointing, especially in regards to the studies investigating primary prevention. Namely, each study has several limitations to test therapeutic agents in clinical practice, mainly because of their low accessibility to tissue or rapid clearance from the body!1,12

In contrast, edaravone (3-methyl-1-phenyl-2-pyrazolin-5one), has a low molecular weight (174.20 kD), is lipophilic, and is readily accessible to tissue! 13,14 Edaravone has the ability to scavenge toxic free radicals; moreover, it has been shown to inhibit vascular endothelial cell injury and to inhibit the aggravation of brain edema caused by free radical-induced lipid peroxidation 15 Recently, edaravone has been officially approved for the treatment of acute ischemic stroke in Japan!6 Previous experimental studies have reported that the administration of edaravone just before reperfusion reduced reperfusion injuries and myocardial damage in myocardial ischemia-reperfusion animal models ${ }^{17,18}$ Edaravone has been shown to attenuate pressure overload-induced LV hypertrophy via its antioxidant function according to a recent report $!^{9}$ With these effects, edaravone has been recognized as both a potent cardioprotective and a cytoprotective agent. We have recently demonstrated that the administration of edaravone just prior to myocardial reperfusion attenuated both enzymatic infarct size and reperfusion arrhythmia in patients with AMI; ${ }^{20}$ however, its effects on long-term clinical outcomes in AMI patients have not yet been clarified. The purpose of the present study was to clarify the efficacy of edaravone on the long-term prognosis in patients with AMI. 


\section{Methods}

\section{Patients and Study Design}

The present study was a randomized, placebo-controlled, open-label study involving 104 patients with initial AMI admitted to our institution from April 2001 to July 2004, who fulfilled the following inclusion criteria: (1) admission within $6 \mathrm{~h}$ of symptom onset; (2) coronary angiography performed immediately after admission; and (3) emergency percutaneous coronary intervention (PCI). The diagnosis of AMI was made on the basis of chest pain persisting for $\geq 30 \mathrm{~min}$, ST-segment elevation of $>0.2 \mathrm{mV}$ in $\geq 2$ contiguous leads on a standard 12-lead electrocardiogram (ECG), and elevation of the serum creatine kinase (CK)-MB isoenzyme level to more than twice the upper limit of the normal level. During the study period, 104 patients, all of whom had had an occluded culprit lesion in the proximal segment of a major coronary artery that persisted after an intra-coronary nitrate injection, were initially enrolled. Of these, 101 patients were randomly divided into either an edaravone group ( $n=50,40$ men; mean $63 \pm 2$ years) or a placebo group ( $n=51,35$ men; mean $63 \pm 2$ years). On admission, we excluded 2 patients with renal insufficiency defined as serum creatinine $>1.2 \mathrm{mg} / \mathrm{dl}$; and we also excluded 1 patient with altered hepatic function, defined as serum asparate aminotransferase $>50 \mathrm{IU} / \mathrm{L}$, alanine aminotransferase $>50 \mathrm{IU} / \mathrm{L}$, and total bilirubin $>1.2 \mathrm{mg} / \mathrm{dl}$. In the edaravone group, $30 \mathrm{mg}$ edaravone was intravenously infused for $10 \mathrm{~min}$ as soon as we confirmed their infarct-related coronary arteries were occluded during coronary angiography; the saline solution was administered as a placebo in the placebo group. The study protocol was in agreement with the guidelines of the Ethics Committee at our institution, and written informed consent was obtained from each patient before they were enrolled in the present study.

\section{Reperfusion Therapy}

Primary PCI was performed instantly and we thereby obtained coronary reperfusion immediately after we confirmed completion of edaravone or placebo infusion. Pre-hospital thrombolysis, distal protection devices and intracoronary thrombolysis, which have been shown to have some efficacy with regards to the prognosis of AMI, were not performed in the present study. In all patients, we succeeded in implanting coronary stents at the culprit lesion to prevent acute coronary occlusion, which is likely to occur after balloon angioplasty alone. The use of concomitant medication was comparable between the groups. Patients were routinely treated with aspirin (100-200 mg/day, indefinitely) and ticlopidine ( $200 \mathrm{mg} /$ day for 2 weeks after coronary stenting). In both groups, an initial bolus of $130 \mathrm{U} / \mathrm{kg}$ heparin was given; additional boluses of heparin were administered during the procedure to maintain an activated clotting time of $300 \mathrm{~s}$.

\section{Determination of Infarct Size and Monitoring of \\ Reperfusion Arrhythmias}

Blood samples were obtained before and immediately after reperfusion, then at $6,12,18,24 \mathrm{~h}$ and at 3, 5, 7 and 14 days after reperfusion. Serum biomarkers were measured by using a standard enzymatic method. We monitored ECGs continuously and compared the incidence of reperfusion arrhythmia such as ventricular tachycardia or ventricular fibrillation. Ventricular tachycardia was defined as $\geq 6$ consecutive premature ventricular contractions.
Incidence of New $Q$ Wave

In recent years the presence or absence of a $Q$ wave has also been used as a surrogate marker to determine infarct size. In the GUSTO angiographic study, in the early convalescent period after fibrinolytic therapy, the $20 \%$ of patients who did not develop Q waves had better global and regional LV function as well as improved 2-year survival rates? We compared the incidence of a new abnormal Q wave in $\geq 2$ contiguous ECG leads at 2 weeks after reperfusion therapy between the 2 groups.

\section{Long-Term Follow-up}

Clinical long-term follow-up was performed via a medical examination at our clinic or telephone contact. Information about potential cardiovascular events was confirmed by source data, including charts of hospital stays, discharge letters, analysis of coronary angiograms or ECGs, and laboratory data. We defined cardiovascular events as any of the following: (1) cardiac death; (2) non-fatal myocardial reinfarction; (3) refractory angina pectoris; (4) ischemic stroke; or (5) heart failure requiring admission. Cardiac death was defined as death from myocardial infarction (MI) or documented sudden death. Myocardial reinfarction was defined as follows: (1) prolonged chest pain (>30 min); (2) new ECG changes (including ischemic ST-segment elevation or depression or pathologic Q waves); (3) increased $\mathrm{CK}-\mathrm{MB}$ isoenzyme and total CK levels more than twice the upper limit of normal. Refractory angina pectoris was defined as exertional angina, angina at rest, or both, which could not be controlled with a combination of antianginal drugs, thereby requiring hospitalization. Ischemic stroke was diagnosed based upon the presence of a neurologic deficit, as confirmed by computed tomography or magnetic resonance imaging.

\section{Serial Determination of Oxidative Stress Markers}

Thioredoxin is a stress-inducible protein, the expression of which is increased by various types of stress, including oxidative stress 22,23 A previous study by Nakamura et al has shown that plasma/serum levels of thioredoxin are elevated under oxidative-stress-associated disorders such as viral infections or ischemia-reperfusion ${ }^{24}$ Recently, human thioredoxin has been quantitatively measured as a marker of oxidative stress by a sandwich enzyme-linked immunosorbent assay (ELISA)? 24,25

In the present study, 33 patients, who agreed to give additional blood samples, had serum thioredoxin levels examined to verify the effect of edaravone as a free radical scavenger in patients with AMI. A sandwich ELISA method for human thioredoxin was provided by FujiRebio (Tokyo, Japan) and used as described in previous studies.24-27

\section{Statistical Analysis}

All data are expressed as mean \pm SEM. Comparisons of age, serum cholesterol, triglyceride and uric acid between the 2 groups were performed with a 1-way analysis of variance followed by Scheffé's test, and the other baseline characteristics were compared by using the chi-square test. We plotted cumulative event curves using Kaplan-Meier survival methods analysis and tested differences between the curves for statistical significance using log-rank analysis. A Cox proportional hazards regression analysis was performed to identify independent predictors of cardiovascular events. $\mathrm{P}$ values $<0.05$ were considered to be statistically significant. 
Table 1 Baseline Characteristics

\begin{tabular}{|c|c|c|c|}
\hline Characteristics & $\begin{array}{c}\text { Edaravone } \\
\text { group } \\
(n=50)\end{array}$ & $\begin{array}{c}\text { Placebo } \\
\text { group } \\
(n=51)\end{array}$ & $p$ value \\
\hline Age (years) & $63 \pm 2$ & $63 \pm 2$ & $N S$ \\
\hline Male gender & $40(80 \%)$ & $35(69 \%)$ & $N S$ \\
\hline Pre-infarction angina & $15(30 \%)$ & $19(37 \%)$ & $N S$ \\
\hline Anterior $M I$ & $30(60 \%)$ & $26(51 \%)$ & $N S$ \\
\hline \multicolumn{4}{|c|}{ Killip classification at admission } \\
\hline$I$ & $42(84 \%)$ & $43(84 \%)$ & $N S$ \\
\hline$I I-I I I$ & $8(16 \%)$ & $8(16 \%)$ & NS \\
\hline \multicolumn{4}{|c|}{ Forrester's classification at admission } \\
\hline Subset I & $19(38 \%)$ & $21(41 \%)$ & NS \\
\hline Subset II-IV & $31(62 \%)$ & $30(59 \%)$ & NS \\
\hline Systemic hypertension & $32(64 \%)$ & $30(59 \%)$ & $N S$ \\
\hline Diabetes mellitus & $14(28 \%)$ & $14(27 \%)$ & $N S$ \\
\hline Cigarette smoking & $31(62 \%)$ & $32(63 \%)$ & $N S$ \\
\hline Body mass index $>26 \mathrm{~kg} / \mathrm{m}^{2}$ & $12(24 \%)$ & $11(22 \%)$ & $N S$ \\
\hline Total cholesterol (mg/dl) & $211 \pm 5$ & $212 \pm 6$ & $N S$ \\
\hline HDL-cholesterol ( $\mathrm{mg} / \mathrm{dl})$ & $50 \pm 2$ & $50 \pm 2$ & $N S$ \\
\hline LDL-cholesterol (mg/dl) & $125 \pm 5$ & $130 \pm 5$ & $N S$ \\
\hline Triglycerides $(\mathrm{mg} / \mathrm{dl})$ & $177 \pm 11$ & $162 \pm 13$ & $N S$ \\
\hline $\operatorname{Uric}$ acid $(m g / d l)$ & $5.6 \pm 0.2$ & $5.7 \pm 0.2$ & $N S$ \\
\hline
\end{tabular}

Values represent number of patients.

MI, myocardial infarction; HDL, high-density lipoprotein; $L D L$, low-density lipoprotein.

Other continuous variables represent mean \pm SEM.

Table 2 Comparison of Clinical Characteristics

\begin{tabular}{|c|c|c|c|}
\hline & $\begin{array}{c}\text { Edaravone } \\
\text { group } \\
(n=50)\end{array}$ & $\begin{array}{c}\text { Placebo } \\
\text { group } \\
(n=51)\end{array}$ & $p$ value \\
\hline \multicolumn{4}{|l|}{ No. of narrowed coronary arteries } \\
\hline 1 & $23(46 \%)$ & $21(41 \%)$ & NS \\
\hline 2 & $18(36 \%)$ & $19(37 \%)$ & NS \\
\hline 3 & $9(18 \%)$ & $11(22 \%)$ & NS \\
\hline \multicolumn{4}{|l|}{ Concomitant medication } \\
\hline ACE inhibitors & $49(98 \%)$ & $48(94 \%)$ & NS \\
\hline Aspirin & $50(100 \%)$ & $51(100 \%)$ & $N S$ \\
\hline Ticlopidine & $50(100 \%)$ & $51(100 \%)$ & $N S$ \\
\hline Statins & $25(50 \%)$ & $18(35 \%)$ & $N S$ \\
\hline$\beta$-blockers & $13(26 \%)$ & $18(35 \%)$ & $N S$ \\
\hline Calcium channel blockers & $40(80 \%)$ & $38(75 \%)$ & $N S$ \\
\hline \multicolumn{4}{|l|}{ Reperfusion procedures } \\
\hline Primary PCI (stenting) & $50(100 \%)$ & $51(100 \%)$ & NS \\
\hline Achievement of $T I M I \geq 3$ & $50(100 \%)$ & $51(100 \%)$ & $N S$ \\
\hline $\begin{array}{l}\text { Duration from onset to } \\
\text { reperfusion }(h)\end{array}$ & $3.5 \pm 0.3$ & $3.4 \pm 0.5$ & $N S$ \\
\hline Peak creatine kinase (IU/L) & $2,246 \pm 221$ & $3,037 \pm 290$ & 0.035 \\
\hline Peak CK-MB isoenzyme (IU/L) & $146 \pm 13$ & $192 \pm 17$ & 0.048 \\
\hline Reperfusion arrhythmias & $1(2 \%)$ & $8(16 \%)$ & 0.031 \\
\hline $\begin{array}{l}\text { Presence of new abnormal } \\
Q \text { wave }\end{array}$ & $23(46 \%)$ & $34(67 \%)$ & 0.046 \\
\hline
\end{tabular}

Values represent mean \pm SEM or number.

$A C E$, angiotensin-converting enzyme; $P C I$, percutaneous coronary intervention; TIMI, Thrombolysis In Myocardial Infarction; $C K$, creatine kinase.

\section{Results}

\section{Comparison of Clinical Characteristics}

The baseline clinical characteristics of the present study subjects are shown in Table 1 . There were no significant differences in age, male gender, pre-infarction angina, the rate of anterior MI, coronary risk factor, or lipid profile between the 2 groups. Eighty-four percent of the patients in both groups were in Killip class I on admission. There were no significant differences in ischemic duration between the
Table 3 Long-Term Clinical Outcomes

\begin{tabular}{lcc}
\hline \hline & $\begin{array}{c}\text { Edaravone } \\
\text { group } \\
(n=50)\end{array}$ & $\begin{array}{c}\text { Placebo } \\
\text { group } \\
(n=51)\end{array}$ \\
\hline Refractory angina pectoris & 1 & 5 \\
Non-fatal MI & 0 & 2 \\
Ischemic stroke & 0 & 1 \\
Cardiac death & 0 & 0 \\
Heart failure requiring admission & 0 & 0 \\
\hline
\end{tabular}

Values represent number of patients. MI, myocardial infarction.

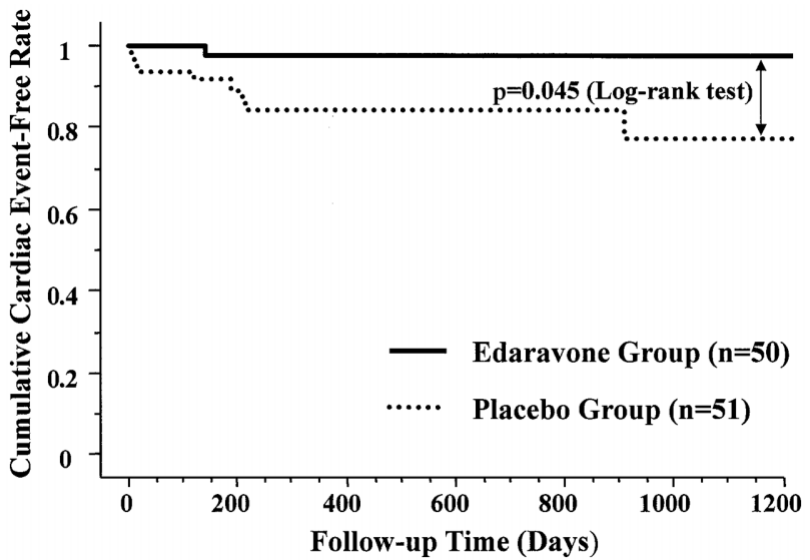

Fig 1. Kaplan-Meier analysis on clinical outcomes between edaravone $(n=50)$ and placebo $(n=51)$ groups. Log-rank $p=0.045$ vs placebo group.

Table 4 Multivariate Predictors of Cardiovascular Event

\begin{tabular}{lcc}
\hline \hline Variables & $\mathrm{X}^{2}$ & $p$ value \\
\hline Absence of edaravone use & 4.312 & 0.038 \\
Ejection fraction $<56 \%$ & 3.691 & 0.055 \\
Cigarette smoking & 3.462 & 0.063 \\
Anterior MI & 2.826 & 0.093 \\
Multivessel disease & 2.742 & 0.098 \\
Hyperuricemia & 2.372 & 0.124 \\
Family history of CVD & 2.310 & 0.129 \\
Absence ofß -blocker use & 2.263 & 0.133 \\
C-reactive protein & 1.707 & 0.191 \\
Hypertension & 1.520 & 0.218 \\
Hyperlipidemia & 1.476 & 0.224 \\
Diabetes mellitus & 1.465 & 0.226 \\
Max. CK-MB isoenzyme $>$ 144 IU/L & 1.226 & 0.268 \\
Duration from onset to reperfusion & 0.662 & 0.416 \\
Absence of statin use & 0.485 & 0.486 \\
Absence of Ca-channel blocker use & 0.282 & 0.596 \\
Male gender & 0.026 & 0.872 \\
Age $>$ 63 years & 0.005 & 0.946
\end{tabular}

MI, myocardial infarction; $C V D$, cerebrovascular disease; $C K$, creatine kinase.

2 groups $(3.5 \pm 0.3 \mathrm{~h}$ in the edaravone group, and $3.4 \pm 0.5 \mathrm{~h}$ in the placebo group; $\mathrm{p}=\mathrm{NS}$ ), and in all cases, the primary PCI was performed against a culprit lesion, and successful reperfusion (defined as the achievement of Thrombolysis In Myocardial Infarction (TIMI) ${ }^{28}$ grade 3 flow) was obtained within $6 \mathrm{~h}$ of symptom onset in all cases. Although a large variety of bare metal stents were used, the frequency in the use of each stent type was comparable between the groups. There were no significant differences in pharmacotherapy, including angiotensin-converting enzyme (ACE) inhibitor 


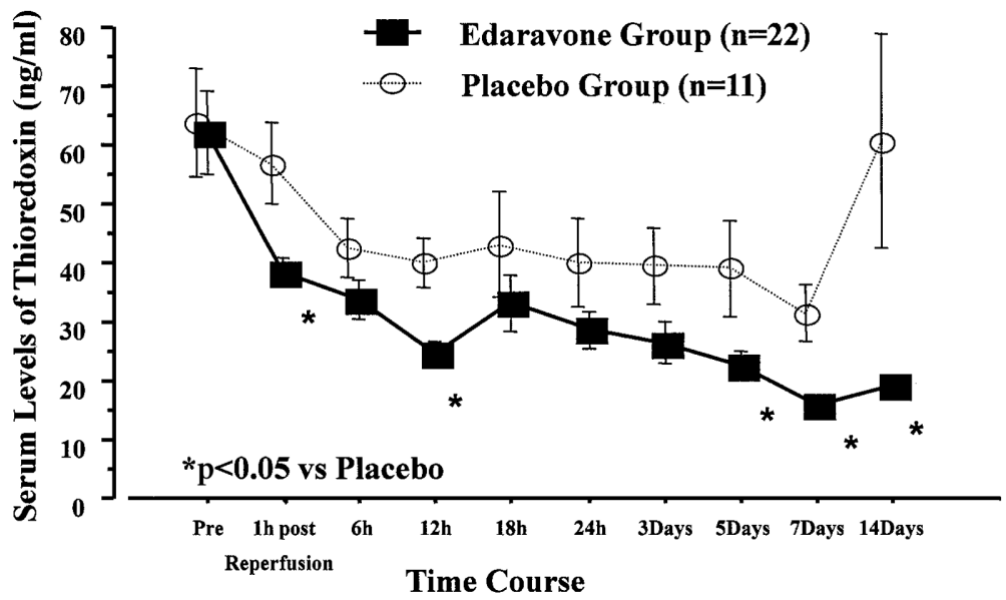

Fig 2. Serial changes in serum thioredoxin levels in the edaravone $(n=22)$ and in the placebo $(n=11)$ groups. ${ }^{*} \mathrm{p}<0.05$ vs placebo group. or $\beta$-blocker use between the 2 groups. In the edaravone group, no patients suffered any adverse effects from the use of edaravone, such as renal insufficiency or altered hepatic function. In addition, there were no significant differences not only in terms of ischemic duration but also in terms of the distribution of infarct-related arteries between the 2 groups.

\section{Comparison of Enzymatic Infarct Size and Reperfusion Arrhythmias}

As we have already reported, the short-term effects of edaravone on infarct size and reperfusion arrhythmias were established again in the present study with a larger sample size than in our previous study? 20 The distribution of infarctrelated occluded arteries and hemodynamic parameters were similar in the 2 groups (Table 1 ). Although there were no differences in the duration of ischemia from symptom onset to reperfusion between the 2 groups, the peak levels of $\mathrm{CK}-\mathrm{MB}$ and $\mathrm{CK}$ were significantly lower in the edaravone group than in the control group, as shown in Table 2 (146 \pm 13 vs $192 \pm 17$ IU/L, $p=0.048$ in CK-MB; and 2,246 \pm 221 vs $3,037 \pm 290 \mathrm{IU} / \mathrm{L}, \mathrm{p}=0.035$ in $\mathrm{CK}$ ).

In contrast, during the first $30 \mathrm{~min}$ after the coronary reperfusion, 8 patients had reperfusion arrhythmia such as ventricular tachycardia or ventricular fibrillation in the placebo group, while only 1 patient had reperfusion arrhythmia in the edaravone group $(\mathrm{p}=0.031)$.

\section{Incidence of a New $Q$ Wave}

As shown in Table 2, although abnormal Q waves were not observed on admission in any subjects, the incidence of a new $Q$ wave was significantly lower in the edaravone group than in the placebo group 2 weeks after reperfusion $(\mathrm{p}=0.046)$.

\section{Long-Term Follow-up and Prognosis of Patients}

The mean follow-up period for all patients was $415 \pm 32$ days (range, 4-1,185 days). In this analysis of long-term clinical outcomes (Table 3 ), although only 1 patient experienced cardiovascular events (refractory angina pectoris) in the edaravone group, 8 patients experienced cardiovascular events ( 5 patients, refractory angina pectoris; and 2 patients, non-fatal MI; and 1 patient, ischemic stroke) in the placebo group. Almost all 7 patients, the ischemic cardiac events in the placebo group were caused by a total or sub-total occlusion based on the progression of coronary stenosis in an infarct-related artery. No instances of cardiac death and heart failure were observed during the study period. Fig 1 shows cardiovascular event-free curves analyzed by KaplanMeier method, and demonstrates that the cardiovascular event-free rates were comparatively high in both study groups; furthermore, log-rank analysis revealed that the event-free rate was significantly higher in the edaravone group than in the placebo group $(\mathrm{p}=0.045)$. Table 4 shows the results of multivariate Cox proportional hazards model analysis for cardiovascular events; the absence of edaravone use was the only independent predictor of cardiovascular events $(\mathrm{p}=0.038)$.

\section{Serum Thioredoxin Levels}

There were no differences in serum thioredoxin levels on admission between the 2 groups; however, the serum thioredoxin levels after reperfusion therapy were significantly lower in the edaravone group than in the placebo group. As shown in Fig 2, especially in the subacute phase, serum thioredoxin levels remained at lower levels in the edaravone group than in the placebo group.

\section{Discussion}

We have previously reported on the short-term efficacy of edaravone for reperfusion injury in patients with AMI and demonstrated that its administration before reperfusion reduces enzymatic infarct size and the incidence of reperfusion arrhythmias ${ }^{20}$ In addition, edaravone suppresses the deterioration of the LV ejection fraction (LVEF) immediately after reperfusion therapy. In other words, edaravone administration could inhibit almost all of the clinical manifestations of reperfusion injury, including myocardial stunning; however, there were no significant differences in LVEF at 2 weeks after reperfusion between the 2 groups, although LVEF in the edaravone group still tended to be higher.

In the present study, all patients received successful reperfusion with TIMI grade 3 flow by primary PCI within a few hours from the onset of symptoms, and were taking ticlopidine as well as aspirin; almost all were taking ACE inhibitors (98\% in the edaravone group; $94 \%$ in the placebo group). It is possible, therefore, that these extremely effective modern therapies may have negated any possible significant differences in LVEF between the 2 groups at 2 weeks after the reperfusion therapy. It is also possible that LVEF, as assessed by echocardiography, might not have been sensitive enough to detect any significant differ- 
ences between the 2 groups in our previous study. In the present study, we compared not only serum cardiac markers, but also the presence of $\mathrm{Q}$ waves to reassess the effect of edaravone on preserved LV function in the convalescent stage. The incidence of a new $Q$ wave was significantly lower in the edaravone group compared with the placebo group. This result could also dovetail with the concept that administration of edaravone on reperfusion might decrease infarct size in patients with AMI. Edaravone administration might be associated with well-preserved LV function as it actually prevents new Q-wave formation.

In contrast, another study conducted by Rajesh et al has recently reported that pretreatment with edaravone before prolonged ischemia prevents the opening of the mitochondrial permeability transition pores by inhibiting the cellular $\mathrm{Ca}^{2+}$ overload because of oxidative stress and thereby reduces necrotic cell death in an experimental model 29 Mitochondria are not only the enzymatic sources of ROS, but they are also a major target for ROS-mediated damage. Mitochondrial ROS generation may lead to a vicious cycle of mitochondrial dysfunction and additional ROS generation, which may play an important role in the development and progression of $\mathrm{LV}$ remodeling and failure ${ }^{30,31}$ It is quite possible that edaravone may attenuate this vicious circle by preventing the opening of the mitochondrial permeability transition pores. In the clinical setting, we have previously investigated the correlation between ROS generation and coronary heart disease ${ }^{32-35}$ Our previous studies have demonstrated that oxidative stress markers, including plasma thioredoxin levels, urinary biopyrrins and 8-hydroxy-2'deoxyguanosine excretion, were elevated in patients with AMI. We have previously reported that increased ROS may predict subsequent cardiovascular events; however, it was still unknown whether edaravone administration reduces ROS generation during the clinical course of AMI in humans. In the present study, serum thioredoxin levels were attenuated just after reperfusion therapy in the edaravone group. In the subacute phase, significantly lower serum thioredoxin levels were maintained at a constant level in the edaravone group but not in the placebo group. The suppressing effect of edaravone on oxidative stress markers in the subacute phase seems not to be caused by the direct effect of edaravone, but by the indirect effects such as the infarct size reducing effect and the anti-inflammatory effect. Therefore, it is suggested that edaravone not only demonstrates a free radical scavenging effect by preventing the vicious circle of ROS generation, but also pleiotropic effects as a cardio-protective agent.

Kaplan-Meier analysis revealed that the cumulative event-free rate in the edaravone group was significantly higher than in the placebo group, even though the rate was comparatively high in both groups. In the multivariate analysis, the absence of edaravone use was the only independent predictor of cardiovascular events. In spite of these modern therapies, it appears that the administration of edaravone provides an additional positive effect and thereby it leads to improved long-term clinical outcomes. Recently, another study suggested that edaravone administration improved endothelial-dependent vasodilation through a decrease in ROS 36 In addition, Janiszewski et al reported that transient increased ROS early after vascular balloon injury markedly amplified the late cellular response and induced a marked increase in neointimal thickening in chronic phase through a vascular redox signaling network 37 Taking into consideration the fact that most of the ischemic cardiac events in the placebo group were based on the progression of coronary stenosis in the infarct-related arteries, the improvement in clinical outcome by edaravone may be due to its pleiotropic effects such as preservation of endothelial function and inhibition of neointimal proliferation.

Edaravone is therefore a useful cardio-protective agent which leads to good clinical outcomes; it does so by scavenging ROS excessively generated during the clinical course of AMI.

These findings provide additional evidence that oxidative stress may play an important role in the pathogenesis of reperfusion injury and that oxidative stress may contribute to cardiac events after reperfusion therapy. In conclusion, intravenous administration of edaravone just before reperfusion may reduce oxidative stress and improve the clinical outcome of AMI patients, in addition to its short-term effects on reperfusion injury.

\section{Acknowledgments}

This study was supported in part by a Grant from the Japan Heart Foundation, a Research Grant for Cardiovascular Disease (14C-4 and 17C-2) from the Ministry of Health, Labor and Welfare, and a Grant-in-Aid for Scientific Research (B-17390232, C-16590698 and C-17590752) from the Ministry of Education, Culture, Sports, Science and Technology in Japan.

\section{References}

1. McCord JM. Oxygen-derived free radicals in post-ischemic injury. $N$ Engl J Med 1985; 312: 159-163.

2. Flaherty JT. Myocardial injury mediated by oxygen free radicals. Am J Med 1991; 91: 3C79S-3C85S.

3. Tsutsui H. Novel pathophysiological insight and treatment strategies for heart failure. Circ J 2004; 68: 1095-1103.

4. Heitzer T, Schlinzig T, Krohn K, Meinertz T, Munzel T. Endothelial dysfunction, oxidative stress, and risk of cardiovascular events in patients with coronary artery disease. Circulation 2001; 104: 2673 2678.

5. Jolly SR, Kane WJ, Bailie MB, Abrams GD, Lucchesi BR. Canine myocardial reperfusion injury: Its reduction by the combined administration of superoxide dismutase and catalase. Circ Res 1984; 54: $277-285$.

6. Altavilla D, Bazzani C, Squadrito F, Cainazzo MM, Mioni C, Bertolini A, et al. IRFI 042, a novel dual vitamin E-like antioxidant, inhibits activation of nuclear factork $\mathrm{B}$ and reduces the inflammatory response in myocardial ischemia-reperfusion injury. Cardiovasc Res 2000; 47: 515-528.

7. Stephens NG, Parsons A, Schofield PM, Kelly F, Cheeseman K, Mitchinson MJ. Randomised controlled trial of vitamin E in patients with coronary disease: Cambridge Heart Antioxidant Study (CHAOS). Lancet 1996; 347: 781-786.

8. Rapola JM, Virtamo J, Ripatti S, Huttunen JK, Albanes D, Taylor PR, et al. Randomised trial of alpha-tocopherol and beta-carotene supplements on incidence of major coronary events in men with previous myocardial infarction. Lancet 1997; 349: 1715-1720.

9. GISSI-Prevenzione Investigators. Dietary supplementation with n-3 polyunsaturated fatty acids and vitamin E after myocardial infarction: Results of the GISSI-Prevenzione trial. Lancet 1999; 354: $447-$ 455.

10. The Heart Outcomes Prevention Evaluation Study Investigators. Vitamin E supplementation and cardiovascular events in high-risk patients. N Engl J Med 2000; 342: 154-160.

11. Rice-Evans CA, Diplock AT. Current status of antioxidant therapy. Free Radic Biol Med 1993; 15: 77-96.

12. Bulkley GB. Free radicals and other reactive oxygen metabolites: Clinical relevance and the therapeutic efficacy of antioxidants therapy. Surgery 1993; 113: 479-483.

13. Nishi H, Watanabe T, Sakurai H, Yuki S, Ishibashi A. Effect of MCI-186 on brain edema in rats. Stroke 1989; 20: 1236-1240.

14. Abe K, Yuki S, Kogure K. Strong attenuation of ischemic and postischemic brain edema in rats by a novel free radical scavenger. Stroke 1988; 19: 480-485.

15. Watanabe T, Egawa M. Effect of an antistroke agent MCI-186 on cerebral arachidonate cascade. J Pharmacol Exp Ther 1994; 271: $1624-1629$.

16. The Edaravone Acute Brain Infarction Study Group. Effect of a 
novel free radical scavenger, edaravone (MCI-186), on acute brain infarction: Randomized, placebo-controlled, double-blind study at multicenters. Cerebrovasc Dis 2003; 15: 222-229.

17. Minhaz U, Tanaka M, Tsukamoto H, Watanabe K, Koide S, Shohtsu A, et al. Effect of MCI-186 on postischemic reperfusion injury in isolated rat heart. Free Radic Res 1996; 24: 361-367.

18. Wu TW, Zeng LH, Wu J, Fung KP. Myocardial protection of MCI186 in rabbit ischemia-reperfusion. Life Sci 2002; 71: 2249-2255.

19. Tsujimoto I, Hikoso S, Yamaguchi O, Kashiwase K, Nakai A, Takeda $\mathrm{T}$, et al. The antioxidant edaravone attenuates pressure overload-induced left ventricular hypertrophy. Hypertension 2005; 45: $921-$ 926.

20. Tsujita K, Shimomura H, Kawano H, Hokamaki J, Fukuda M, Yamashita T, et al. Effects of edaravone on reperfusion injury in patients with acute myocardial infarction. Am J Cardiol 2004; 94: 481 484.

21. Goodman SG, Langer A, Ross AM, Wildermann NM, Barbagelata A, Sgarbossa EB, et al. Non-Q-wave versus Q-wave myocardial infarction after thrombolytic therapy: Angiographic and prognostic insights from the global utilization of streptokinase and tissue plasminogen activator for occluded coronary arteries-I angiographic substudy: GUSTO-I Angiographic Investigators. Circulation 1998; 97: $444-$ 450.

22. Nakamura H, Matsuda M, Furuke K, Kitaoka Y, Iwata S, Toda K, et al. Adult T cell leukemia-derived factor/human thioredoxin protects endothelial F-2 cell injury caused by activated neutrophils or hydrogen peroxide. Immunol Lett 1994; 42: 75-80.

23. Ohira A, Honda O, Gauntt CD, Yamamoto M, Hori K, Masutani H, et al. Oxidative stress induces adult $\mathrm{T}$ cell leukemia derived factor/thioredoxin in the rat retina. Lab Invest 1994; 70: 279-285.

24. Nakamura H, De Rosa S, Roederer M, Anderson MT, Dubs JG, Yodoi J, et al. Elevation of plasma thioredoxin levels in HIV-infected individuals. Int Immunol 1996; 8: 603-611.

25. Miwa K, Kishimoto C, Nakamura H, Makita T, Ishii K, Okuda N, et al. Serum thioredoxin and a-tocopherol concentrations in patients with major risk factors. Circ J 2005; 69: 291-294.

26. Kawano H, Yasue H, Hirai N, Yoshida T, Fukushima H, Miyamoto $\mathrm{S}$, et al. Effects of transdermal and oral estrogen supplementation on endothelial function, inflammation and cellular redox state. Int J Clin Pharmacol Ther 2003; 41: 346-353.

27. Hirai N, Kawano H, Yasue H, Shimomura H, Miyamoto S, Soejima
$\mathrm{H}$, et al. Attenuation of nitrate tolerance and oxidative stress by an angiotensin II receptor blocker in patients with coronary spastic angina. Circulation 2003; 108: $1446-1450$.

28. The TIMI Study Group. The thrombolysis in myocardial infarction (TIMI) trial: Phase 1 findings. N Engl J Med 1985; 312: 932-936.

29. Rajesh KG, Sasaguri S, Suzuki R, Maeda H. Antioxidant MCI-186 inhibits mitochondrial permeability transition pore and upregulates Bcl-2 expression. Am J Physiol Heart Circ Physiol 2003; 285: $\mathrm{H} 2171-\mathrm{H} 2178$.

30. Ide T, Tsutsui H, Hayashidani S, Kang D, Suematsu N, Nakamura K, et al. Mitochondrial DNA damage and dysfunction associated with oxidative stress in failing hearts after myocardial infarction. Circ Res 2001; 88: 529-535.

31. Tsutsui H, Ide T, Shiomi T, Kang D, Hayashidani S, Suematsu N, et al. 8-Oxo-dGTPase, which prevents oxidative stress-induced DNA damage, increases in the mitochondria from failing hearts. Circulation 2001; 104: 2883-2885.

32. Shimomura H, Ogawa H, Takazoe K, Soejima H, Miyamoto S, Sakamoto T, et al. Comparison of urinary biopyrrin levels in acute myocardial infarction (after reperfusion therapy) versus stable angina pectoris and their usefulness in predicting subsequent cardiac events. Am J Cardiol 2002; 90: 108-111.

33. Miyamoto S, Sakamoto T, Soejima H, Shimomura H, Kajiwara I, Kojima S, et al. Plasma thioredoxin levels and platelet aggregability in patients with acute myocardial infarction. Am Heart $J$ 2003; 146: $465-471$.

34. Soejima H, Suefuji H, Miyamoto S, Kajiwara I, Kojima S, Hokamaki $\mathrm{J}$, et al. Increased plasma thioredoxin in patients with acute myocardial infarction. Clin Cardiol 2003; 26: 583-587.

35. Nagayoshi Y, Kawano H, Hokamaki J, Miyamoto S, Kojima S, Shimomura H, et al. Urinary 8-hydroxy-2'-deoxyguanosine levels increase after reperfusion in acute myocardial infarction and may predict subsequent cardiac events. Am J Cardiol 2005; 95: 514-517.

36. Jitsuiki D, Higashi Y, Goto C, Kimura M, Noma K, Hara K, et al. Effect of edaravone, a novel free radical scavenger, on endotheliumdependent vasodilation in smokers. Am J Cardiol 2004; 94: 10701073 .

37. Janiszewski M, Pasqualucci CA, Souza LC, Pileggi F, da Luz PL, Laurindo FR. Oxidized thiols markedly amplify the vascular response to balloon injury in rabbits through a redox active metaldependent pathway. Cardiovasc Res 1998; 39: 327-338. 